\title{
Partial Differentiation of Vector-Valued Functions on $n$-Dimensional Real Normed Linear Spaces
}

\author{
Takao Inoué \\ Inaba 2205, Wing-Minamikan \\ Nagano, Nagano, Japan \\ Adam Naumowicz \\ Institute of Computer Science \\ University of Białystok \\ Akademicka 2, 15-267 Białystok, Poland \\ Noboru Endou \\ Yasunari Shidama \\ Gifu National College of Technology \\ Japan \\ Shinshu University \\ Nagano, Japan
}

\begin{abstract}
Summary. In this article, we define and develop partial differentiation of vector-valued functions on $n$-dimensional real normed linear spaces (refer to [19]
\end{abstract} and $[20])$.

MML identifier: $\underline{\text { PDIFF_7 }}$, version: $\underline{7.11 .07 \quad 4.156 .1112}$

The notation and terminology used in this paper have been introduced in the following papers: [7], [15], [2], [3], [24], [4], [5], [1], [11], [16], [6], [9], [12], [17], [18], [10], [8], [23], [14], [21], [13], and [22].

For simplicity, we use the following convention: $n, m$ denote non empty elements of $\mathbb{N}, i, j$ denote elements of $\mathbb{N}, f$ denotes a partial function from $\left\langle\mathcal{E}^{m},\|\cdot\|\right\rangle$ to $\left\langle\mathcal{E}^{n},\|\cdot\|\right\rangle, g$ denotes a partial function from $\mathcal{R}^{m}$ to $\mathcal{R}^{n}, h$ denotes a partial function from $\mathcal{R}^{m}$ to $\mathbb{R}, x$ denotes a point of $\left\langle\mathcal{E}^{m},\|\cdot\|\right\rangle, y$ denotes an element of $\mathcal{R}^{m}$, and $X$ denotes a set.

We now state a number of propositions: 
(1) If $i \leq j$, then $\langle\underbrace{0, \ldots, 0}_{j}\rangle i i=\langle\underbrace{0, \ldots, 0}_{i}\rangle$.

(2) If $i \leq j$, then $\langle\underbrace{0, \ldots, 0}_{j}\rangle \Gamma\left(i-^{\prime} 1\right)=\langle\underbrace{0, \ldots, 0}_{i-^{\prime} 1}\rangle$.

(3) $\langle\underbrace{0, \ldots, 0}_{j}\rangle_{l i}=\langle\underbrace{0, \ldots, 0}_{j-^{\prime} i}\rangle$.

(4) If $i \leq j$, then $\langle\underbrace{0, \ldots, 0}_{j}\rangle \Gamma\left(i-^{\prime} 1\right)=\langle\underbrace{0, \ldots, 0}_{i-{-^{\prime}}^{1}}\rangle$ and $\langle\underbrace{0, \ldots, 0}_{j}\rangle_{l i}=\langle\underbrace{0, \ldots, 0}_{j-^{\prime} i}\rangle$.

(5) For every element $x_{1}$ of $\left\langle\mathcal{E}^{1},\|\cdot\|\right\rangle$ such that $1 \leq i \leq j$ holds $\left\|\left(\operatorname{reproj}\left(i, 0_{\left\langle\mathcal{E}^{j},\|\cdot\|\right\rangle}\right)\right)\left(x_{1}\right)\right\|=\left\|x_{1}\right\|$.

(6) Let $m, i$ be elements of $\mathbb{N}, x$ be an element of $\mathcal{R}^{m}$, and $r$ be a real number. Then $(\operatorname{reproj}(i, x))(r)-x=(\operatorname{reproj}(i,\langle\underbrace{0, \ldots, 0}_{m}\rangle))(r-(\operatorname{proj}(i, m))(x))$ and $x-(\operatorname{reproj}(i, x))(r)=(\operatorname{reproj}(i,\langle\underbrace{0, \ldots, 0}_{m}\rangle))((\operatorname{proj}(i, m))(x)-r)$.

(7) Let $m, i$ be elements of $\mathbb{N}, x$ be a point of $\left\langle\mathcal{E}^{m},\|\cdot\|\right\rangle$, and $p$ be a point of $\left\langle\mathcal{E}^{1},\|\cdot\|\right\rangle$. Then $(\operatorname{reproj}(i, x))(p)-x=$ $\left(\operatorname{reproj}\left(i, 0_{\left\langle\mathcal{E}^{m},\|\cdot\|\right\rangle}\right)\right)(p-(\operatorname{Proj}(i, m))(x))$ and $x-(\operatorname{reproj}(i, x))(p)=$ $\left(\operatorname{reproj}\left(i, 0_{\left\langle\mathcal{E}^{m},\|\cdot\|\right\rangle}\right)\right)((\operatorname{Proj}(i, m))(x)-p)$.

(8) Let $m, n$ be non empty elements of $\mathbb{N}, i$ be an element of $\mathbb{N}, f$ be a partial function from $\left\langle\mathcal{E}^{m},\|\cdot\|\right\rangle$ to $\left\langle\mathcal{E}^{n},\|\cdot\|\right\rangle$, and $Z$ be a subset of $\left\langle\mathcal{E}^{m},\|\cdot\|\right\rangle$. Suppose $Z$ is open and $1 \leq i \leq m$. Then $f$ is partially differentiable on $Z$ w.r.t. $i$ if and only if $Z \subseteq \operatorname{dom} f$ and for every point $x$ of $\left\langle\mathcal{E}^{m},\|\cdot\|\right\rangle$ such that $x \in Z$ holds $f$ is partially differentiable in $x$ w.r.t. $i$.

(9) For all elements $x, y$ of $\mathbb{R}$ and for every element $i$ of $\mathbb{N}$ such that $1 \leq i \leq m$ holds Replace $(\langle\underbrace{0, \ldots, 0}_{m}\rangle, i, x+y)=\operatorname{Replace}(\langle\underbrace{0, \ldots, 0}_{m}\rangle, i, x)+$ Replace $(\langle\underbrace{0, \ldots, 0}_{m}\rangle, i, y)$.

(10) For all elements $x, a$ of $\mathbb{R}$ and for every element $i$ of $\mathbb{N}$ such that $1 \leq i \leq m$ holds Replace $(\langle\underbrace{0, \ldots, 0}_{m}\rangle, i, a \cdot x)=a \cdot \operatorname{Replace}(\langle\underbrace{0, \ldots, 0}_{m}\rangle, i, x)$.

(11) For every element $x$ of $\mathbb{R}$ and for every element $i$ of $\mathbb{N}$ such that $1 \leq i \leq m$ and $x \neq 0$ holds Replace $(\langle\underbrace{0, \ldots, 0}_{m}\rangle, i, x) \neq\langle\underbrace{0, \ldots, 0}_{m}\rangle$.

(12) Let $x, y$ be elements of $\mathbb{R}, z$ be an element of $\mathcal{R}^{m}$, and $i$ be an element of $\mathbb{N}$. Suppose $1 \leq i \leq m$ and $y=(\operatorname{proj}(i, m))(z)$. Then $\operatorname{Replace}(z, i, x)-z=\operatorname{Replace}(\langle\underbrace{0, \ldots, 0}_{m}\rangle, i, x-y)$ and $z-\operatorname{Replace}(z, i, x)=$ Replace $(\langle\underbrace{0, \ldots, 0}_{m}\rangle, i, y-x)$. 
(13) For all elements $x, y$ of $\mathbb{R}$ and for every element $i$ of $\mathbb{N}$ such that $1 \leq$ $i \leq m$ holds $(\operatorname{reproj}(i,\langle\underbrace{0, \ldots, 0}_{m}\rangle))(x+y)=(\operatorname{reproj}(i,\langle\underbrace{0, \ldots, 0}_{m}\rangle))(x)+$ $(\operatorname{reproj}(i,\langle\underbrace{0, \ldots, 0}_{m}\rangle))(y)$.

(14) For all points $x, y$ of $\left\langle\mathcal{E}^{1},\|\cdot\|\right\rangle$ and for every element $i$ of $\mathbb{N}$ such that $1 \leq i \leq m$ holds $\left(\operatorname{reproj}\left(i, 0_{\left\langle\mathcal{E}^{m},\|\cdot\|\right\rangle}\right)\right)(x+y)=\left(\operatorname{reproj}\left(i, 0_{\left\langle\mathcal{E}^{m},\|\cdot\|\right\rangle}\right)\right)(x)+$ $\left(\operatorname{reproj}\left(i, 0_{\left\langle\mathcal{E}^{m},\|\cdot\|\right\rangle}\right)\right)(y)$.

(15) For all elements $x, a$ of $\mathbb{R}$ and for every element $i$ of $\mathbb{N}$ such that $1 \leq i \leq m$ holds $(\operatorname{reproj}(i,\langle\underbrace{0, \ldots, 0}_{m}\rangle))(a \cdot x)=a \cdot(\operatorname{reproj}(i,\langle\underbrace{0, \ldots, 0}_{m}\rangle))(x)$.

(16) Let $x$ be a point of $\left\langle\mathcal{E}^{1},\|\cdot\|\right\rangle, a$ be an element of $\mathbb{R}$, and $i$ be an element of $\mathbb{N}$. If $1 \leq i \leq m$, then $\left(\operatorname{reproj}\left(i, 0_{\left\langle\mathcal{E}^{m},\|\cdot\|\right\rangle}\right)\right)(a \cdot x)=a \cdot\left(\operatorname{reproj}\left(i, 0_{\left\langle\mathcal{E}^{m},\|\cdot\|\right\rangle}\right)\right)(x)$.

(17) For every element $x$ of $\mathbb{R}$ and for every element $i$ of $\mathbb{N}$ such that $1 \leq i \leq m$ and $x \neq 0$ holds $(\operatorname{reproj}(i,\langle\underbrace{0, \ldots, 0}_{m}\rangle))(x) \neq\langle\underbrace{0, \ldots, 0}_{m}\rangle$.

(18) For every point $x$ of $\left\langle\mathcal{E}^{1},\|\cdot\|\right\rangle$ and for every element $i$ of $\mathbb{N}$ such that $1 \leq i \leq m$ and $x \neq 0_{\left\langle\mathcal{E}^{1},\|\cdot\|\right\rangle}$ holds $\left(\operatorname{reproj}\left(i, 0_{\left\langle\mathcal{E}^{m},\|\cdot\|\right\rangle}\right)\right)(x) \neq 0_{\left\langle\mathcal{E}^{m},\|\cdot\|\right\rangle} \cdot$

(19) Let $x, y$ be elements of $\mathbb{R}, z$ be an element of $\mathcal{R}^{m}$, and $i$ be an element of $\mathbb{N}$. Suppose $1 \leq i \leq m$ and $y=(\operatorname{proj}(i, m))(z)$. Then $(\operatorname{reproj}(i, z))(x)-z=(\operatorname{reproj}(i, \underbrace{0, \ldots, 0}_{m}\rangle))(x-y)$ and $z-$ $(\operatorname{reproj}(i, z))(x)=(\operatorname{reproj}(i,\langle\underbrace{0, \ldots, 0}_{m}\rangle))(y-x)^{m}$.

(20) Let $x, y$ be points of $\left\langle\mathcal{E}^{1},\|\cdot\|\right\rangle, i$ be an element of $\mathbb{N}$, and $z$ be a point of $\left\langle\mathcal{E}^{m},\|\cdot\|\right\rangle$. Suppose $1 \leq i \leq m$ and $y=(\operatorname{Proj}(i, m))(z)$. Then $(\operatorname{reproj}(i, z))(x)-z=\left(\operatorname{reproj}\left(i, 0_{\left\langle\mathcal{E}^{m},\|\cdot\|\right\rangle}\right)\right)(x-y)$ and $z-(\operatorname{reproj}(i, z))(x)=$ $\left(\operatorname{reproj}\left(i, 0_{\left\langle\mathcal{E}^{m},\|\cdot\|\right\rangle}\right)\right)(y-x)$.

(21) Suppose $f$ is differentiable in $x$ and $1 \leq i \leq m$. Then $f$ is partially differentiable in $x$ w.r.t. $i$ and $\operatorname{partdiff}(f, x, i)=f^{\prime}(x) \cdot \operatorname{reproj}\left(i, 0_{\left\langle\mathcal{E}^{m},\|\cdot\|\right\rangle}\right)$.

(22) Suppose $g$ is differentiable in $y$ and $1 \leq i \leq m$. Then $g$ is partially differentiable in $y$ w.r.t. $i$ and partdiff $(g, y, i)=\left(g^{\prime}(y) \cdot \operatorname{reproj}\left(i, 0_{\left\langle\mathcal{E}^{m},\|\cdot\|\right\rangle}\right)\right)(\langle 1\rangle)$.

Let $n$ be a non empty element of $\mathbb{N}$, let $f$ be a partial function from $\mathcal{R}^{n}$ to $\mathbb{R}$, and let $x$ be an element of $\mathcal{R}^{n}$. We say that $f$ is differentiable in $x$ if and only if:

(Def. 1) $\langle f\rangle$ is differentiable in $x$.

Let $n$ be a non empty element of $\mathbb{N}$, let $f$ be a partial function from $\mathcal{R}^{n}$ to $\mathbb{R}$, and let $x$ be an element of $\mathcal{R}^{n}$. The functor $f^{\prime}(x)$ yielding a function from $\mathcal{R}^{n}$ into $\mathbb{R}$ is defined as follows:

(Def. 2) $f^{\prime}(x)=\operatorname{proj}(1,1) \cdot\langle f\rangle^{\prime}(x)$.

Next we state several propositions: 
(23) Suppose $h$ is differentiable in $y$ and $1 \leq i \leq m$. Then $h$ is partially differentiable in $y$ w.r.t. $i$ and $\operatorname{partdiff}(h, y, i)=(h \cdot \operatorname{reproj}(i, y))^{\prime}((\operatorname{proj}(i, m))(y))$ and $\left.\operatorname{partdiff}(h, y, i)=h^{\prime}(y)((\operatorname{reproj}(i, \underbrace{0, \ldots, 0}_{m}\rangle))(1)\right)$.

(24) Let $m$ be a non empty element of $\mathbb{N}$ and $v, w, u$ be finite sequences of elements of $\mathcal{R}^{m}$. If $\operatorname{dom} v=\operatorname{dom} w$ and $u=v+w$, then $\sum u=\sum v+\sum w$.

(25) Let $m$ be a non empty element of $\mathbb{N}, r$ be a real number, and $w, u$ be finite sequences of elements of $\mathcal{R}^{m}$. If $u=r w$, then $\sum u=r \cdot \sum w$.

(26) Let $n$ be a non empty element of $\mathbb{N}$ and $h, g$ be finite sequences of elements of $\mathcal{R}^{n}$. Suppose len $h=\operatorname{len} g+1$ and for every natural number $i$ such that $i \in \operatorname{dom} g$ holds $g_{i}=h_{i}-h_{i+1}$. Then $h_{1}-h_{\operatorname{len} h}=\sum g$.

(27) Let $n$ be a non empty element of $\mathbb{N}$ and $h, g, j$ be finite sequences of elements of $\mathcal{R}^{n}$. Suppose len $h=\operatorname{len} j$ and len $g=\operatorname{len} j$ and for every natural number $i$ such that $i \in \operatorname{dom} j$ holds $j_{i}=h_{i}-g_{i}$. Then $\sum j=$ $\sum h-\sum g$.

(28) Let $m, n$ be non empty elements of $\mathbb{N}, f$ be a partial function from $\mathcal{R}^{m}$ to $\mathcal{R}^{n}$, and $x, y$ be elements of $\mathcal{R}^{m}$. Then there exists a finite sequence $h$ of elements of $\mathcal{R}^{m}$ and there exists a finite sequence $g$ of elements of $\mathcal{R}^{n}$ such that

(i) $\operatorname{len} h=m+1$,

(ii) $\operatorname{len} g=m$,

(iii) for every natural number $i$ such that $i \in \operatorname{dom} h$ holds $h_{i}=(y \uparrow((m+$ 1) $\left.\left.-^{\prime} i\right)\right) \frown\langle\underbrace{0, \ldots, 0}_{i-{ }^{\prime} 1}\rangle$,

(iv) for every natural number $i$ such that $i \in \operatorname{dom} g$ holds $g_{i}=f_{x+h_{i}}-$ $f_{x+h_{i+1}}$,

(v) for every natural number $i$ and for every element $h_{1}$ of $\mathcal{R}^{m}$ such that $i \in \operatorname{dom} h$ and $h_{i}=h_{1}$ holds $\left|h_{1}\right| \leq|y|$, and

(vi) $f_{x+y}-f_{x}=\sum g$.

(29) Let $m$ be a non empty element of $\mathbb{N}$ and $f$ be a partial function from $\mathcal{R}^{m}$ to $\mathcal{R}^{1}$. Then there exists a partial function $f_{0}$ from $\mathcal{R}^{m}$ to $\mathbb{R}$ such that $f=\left\langle f_{0}\right\rangle$.

(30) Let $m, n$ be non empty elements of $\mathbb{N}, f$ be a partial function from $\mathcal{R}^{m}$ to $\mathcal{R}^{n}, f_{0}$ be a partial function from $\left\langle\mathcal{E}^{m},\|\cdot\|\right\rangle$ to $\left\langle\mathcal{E}^{n},\|\cdot\|\right\rangle, x$ be an element of $\mathcal{R}^{m}$, and $x_{0}$ be an element of $\left\langle\mathcal{E}^{m},\|\cdot\|\right\rangle$. If $x \in \operatorname{dom} f$ and $x=x_{0}$ and $f=f_{0}$, then $f_{x}=\left(f_{0}\right)_{x_{0}}$.

Let $m$ be a non empty element of $\mathbb{N}$ and let $X$ be a subset of $\mathcal{R}^{m}$. We say that $X$ is open if and only if:

(Def. 3) There exists a subset $X_{0}$ of $\left\langle\mathcal{E}^{m},\|\cdot\|\right\rangle$ such that $X_{0}=X$ and $X_{0}$ is open.

The following proposition is true 
(31) Let $m$ be a non empty element of $\mathbb{N}$ and $X$ be a subset of $\mathcal{R}^{m}$. Then $X$ is open if and only if for every element $x$ of $\mathcal{R}^{m}$ such that $x \in X$ there exists a real number $r$ such that $r>0$ and $\left\{y \in \mathcal{R}^{m}:|y-x|<r\right\} \subseteq X$.

Let $m, n$ be non empty elements of $\mathbb{N}$, let $i$ be an element of $\mathbb{N}$, let $f$ be a partial function from $\mathcal{R}^{m}$ to $\mathcal{R}^{n}$, and let $X$ be a set. We say that $f$ is partially differentiable on $X$ w.r.t. $i$ if and only if:

(Def. 4) $X \subseteq \operatorname{dom} f$ and for every element $x$ of $\mathcal{R}^{m}$ such that $x \in X$ holds $f\lceil X$ is partially differentiable in $x$ w.r.t. $i$.

One can prove the following propositions:

(32) Let $m, n$ be non empty elements of $\mathbb{N}$ and $f$ be a partial function from $\mathcal{R}^{m}$ to $\mathcal{R}^{n}$. Suppose $f$ is partially differentiable on $X$ w.r.t. $i$. Then $X$ is a subset of $\mathcal{R}^{m}$.

(33) Let $m, n$ be non empty elements of $\mathbb{N}, i$ be an element of $\mathbb{N}, f$ be a partial function from $\mathcal{R}^{m}$ to $\mathcal{R}^{n}, g$ be a partial function from $\left\langle\mathcal{E}^{m},\|\cdot\|\right\rangle$ to $\left\langle\mathcal{E}^{n},\|\cdot\|\right\rangle$, and $Z$ be a set. Suppose $f=g$. Then $f$ is partially differentiable on $Z$ w.r.t. $i$ if and only if $g$ is partially differentiable on $Z$ w.r.t. $i$.

(34) Let $m, n$ be non empty elements of $\mathbb{N}, i$ be an element of $\mathbb{N}, f$ be a partial function from $\mathcal{R}^{m}$ to $\mathcal{R}^{n}$, and $Z$ be a subset of $\mathcal{R}^{m}$. Suppose $Z$ is open and $1 \leq i \leq m$. Then $f$ is partially differentiable on $Z$ w.r.t. $i$ if and only if $Z \subseteq \operatorname{dom} f$ and for every element $x$ of $\mathcal{R}^{m}$ such that $x \in Z$ holds $f$ is partially differentiable in $x$ w.r.t. $i$.

Let $m, n$ be non empty elements of $\mathbb{N}$, let $i$ be an element of $\mathbb{N}$, let $f$ be a partial function from $\mathcal{R}^{m}$ to $\mathcal{R}^{n}$, and let us consider $X$. Let us assume that $f$ is partially differentiable on $X$ w.r.t. $i$. The functor $f \uparrow^{i} X$ yielding a partial function from $\mathcal{R}^{m}$ to $\mathcal{R}^{n}$ is defined as follows:

(Def. 5) $\operatorname{dom}\left(f \uparrow^{i} X\right)=X$ and for every element $x$ of $\mathcal{R}^{m}$ such that $x \in X$ holds $\left(f \uparrow^{i} X\right)_{x}=\operatorname{partdiff}(f, x, i)$.

Let $m, n$ be non empty elements of $\mathbb{N}$, let $f$ be a partial function from $\mathcal{R}^{m}$ to $\mathcal{R}^{n}$, and let $x_{0}$ be an element of $\mathcal{R}^{m}$. We say that $f$ is continuous in $x_{0}$ if and only if:

(Def. 6) There exists a point $y_{0}$ of $\left\langle\mathcal{E}^{m},\|\cdot\|\right\rangle$ and there exists a partial function $g$ from $\left\langle\mathcal{E}^{m},\|\cdot\|\right\rangle$ to $\left\langle\mathcal{E}^{n},\|\cdot\|\right\rangle$ such that $x_{0}=y_{0}$ and $f=g$ and $g$ is continuous in $y_{0}$.

The following propositions are true:

(35) Let $m, n$ be non empty elements of $\mathbb{N}, f$ be a partial function from $\mathcal{R}^{m}$ to $\mathcal{R}^{n}, g$ be a partial function from $\left\langle\mathcal{E}^{m},\|\cdot\|\right\rangle$ to $\left\langle\mathcal{E}^{n},\|\cdot\|\right\rangle, x$ be an element of $\mathcal{R}^{m}$, and $y$ be a point of $\left\langle\mathcal{E}^{m},\|\cdot\|\right\rangle$. Suppose $f=g$ and $x=y$. Then $f$ is continuous in $x$ if and only if $g$ is continuous in $y$.

(36) Let $m, n$ be non empty elements of $\mathbb{N}, f$ be a partial function from $\mathcal{R}^{m}$ to $\mathcal{R}^{n}$, and $x_{0}$ be an element of $\mathcal{R}^{m}$. Then $f$ is continuous in $x_{0}$ if and 
only if the following conditions are satisfied:

(i) $\quad x_{0} \in \operatorname{dom} f$, and

(ii) for every real number $r$ such that $0<r$ there exists a real number $s$ such that $0<s$ and for every element $x_{2}$ of $\mathcal{R}^{m}$ such that $x_{2} \in \operatorname{dom} f$ and $\left|x_{2}-x_{0}\right|<s$ holds $\left|f_{x_{2}}-f_{x_{0}}\right|<r$.

Let $m, n$ be non empty elements of $\mathbb{N}$, let $f$ be a partial function from $\mathcal{R}^{m}$ to $\mathcal{R}^{n}$, and let us consider $X$. We say that $f$ is continuous on $X$ if and only if:

(Def. 7) $X \subseteq \operatorname{dom} f$ and for every element $x_{0}$ of $\mathcal{R}^{m}$ such that $x_{0} \in X$ holds $f\lceil X$ is continuous in $x_{0}$.

Next we state a number of propositions:

(37) Let $m, n$ be non empty elements of $\mathbb{N}, f$ be a partial function from $\mathcal{R}^{m}$ to $\mathcal{R}^{n}, g$ be a partial function from $\left\langle\mathcal{E}^{m},\|\cdot\|\right\rangle$ to $\left\langle\mathcal{E}^{n},\|\cdot\|\right\rangle$, and $X$ be a set. If $f=g$, then $f$ is continuous on $X$ iff $g$ is continuous on $X$.

(38) Let $m, n$ be non empty elements of $\mathbb{N}, f$ be a partial function from $\mathcal{R}^{m}$ to $\mathcal{R}^{n}$, and $X$ be a set. Then $f$ is continuous on $X$ if and only if the following conditions are satisfied:

(i) $X \subseteq \operatorname{dom} f$, and

(ii) for every element $x_{0}$ of $\mathcal{R}^{m}$ and for every real number $r$ such that $x_{0} \in X$ and $0<r$ there exists a real number $s$ such that $0<s$ and for every element $x_{2}$ of $\mathcal{R}^{m}$ such that $x_{2} \in X$ and $\left|x_{2}-x_{0}\right|<s$ holds $\left|f_{x_{2}}-f_{x_{0}}\right|<r$.

(39) Let $m$ be a non empty element of $\mathbb{N}, x, y$ be elements of $\mathcal{R}^{m}, i$ be an element of $\mathbb{N}$, and $x_{1}$ be a real number. If $1 \leq i \leq m$ and $y=(\operatorname{reproj}(i, x))\left(x_{1}\right)$, then $(\operatorname{proj}(i, m))(y)=x_{1}$.

(40) Let $m$ be a non empty element of $\mathbb{N}, f$ be a partial function from $\mathcal{R}^{m}$ to $\mathbb{R}, x, y$ be elements of $\mathcal{R}^{m}, i$ be an element of $\mathbb{N}$, and $x_{1}$ be a real number. If $1 \leq i \leq m$ and $y=(\operatorname{reproj}(i, x))\left(x_{1}\right)$, then $\operatorname{reproj}(i, x)=\operatorname{reproj}(i, y)$.

(41) Let $m$ be a non empty element of $\mathbb{N}, f$ be a partial function from $\mathcal{R}^{m}$ to $\mathbb{R}$, $g$ be a partial function from $\mathbb{R}$ to $\mathbb{R}, x, y$ be elements of $\mathcal{R}^{m}, i$ be an element of $\mathbb{N}$, and $x_{1}$ be a real number. If $1 \leq i \leq m$ and $y=(\operatorname{reproj}(i, x))\left(x_{1}\right)$ and $g=f \cdot \operatorname{reproj}(i, x)$, then $g^{\prime}\left(x_{1}\right)=\operatorname{partdiff}(f, y, i)$.

(42) Let $m$ be a non empty element of $\mathbb{N}, f$ be a partial function from $\mathcal{R}^{m}$ to $\mathbb{R}, p, q$ be real numbers, $x$ be an element of $\mathcal{R}^{m}$, and $i$ be an element of $\mathbb{N}$. Suppose that

(i) $1 \leq i$,

(ii) $i \leq m$,

(iii) $p<q$,

(iv) for every real number $h$ such that $h \in[p, q] \operatorname{holds}(\operatorname{reproj}(i, x))(h) \in$ $\operatorname{dom} f$, and 
(v) for every real number $h$ such that $h \in[p, q]$ holds $f$ is partially differentiable in $(\operatorname{reproj}(i, x))(h)$ w.r.t. $i$.

Then there exists a real number $r$ and there exists an element $y$ of $\mathcal{R}^{m}$ such that $r \in] p, q\left[\right.$ and $y=(\operatorname{reproj}(i, x))(r)$ and $f_{(\operatorname{reproj}(i, x))(q)}-f_{(\operatorname{reproj}(i, x))(p)}=$ $(q-p) \cdot \operatorname{partdiff}(f, y, i)$.

(43) Let $m$ be a non empty element of $\mathbb{N}, f$ be a partial function from $\mathcal{R}^{m}$ to $\mathbb{R}, p, q$ be real numbers, $x$ be an element of $\mathcal{R}^{m}$, and $i$ be an element of $\mathbb{N}$. Suppose that

(i) $1 \leq i$,

(ii) $i \leq m$,

(iii) $p \leq q$,

(iv) for every real number $h$ such that $h \in[p, q] \operatorname{holds}(\operatorname{reproj}(i, x))(h) \in$ $\operatorname{dom} f$, and

(v) for every real number $h$ such that $h \in[p, q]$ holds $f$ is partially differentiable in $(\operatorname{reproj}(i, x))(h)$ w.r.t. $i$.

Then there exists a real number $r$ and there exists an element $y$ of $\mathcal{R}^{m}$ such that $r \in[p, q]$ and $y=(\operatorname{reproj}(i, x))(r)$ and $f_{(\operatorname{reproj}(i, x))(q)}-f_{(\operatorname{reproj}(i, x))(p)}=$ $(q-p) \cdot \operatorname{partdiff}(f, y, i)$.

(44) Let $m$ be a non empty element of $\mathbb{N}, x, y, z, w$ be elements of $\mathcal{R}^{m}, i$ be an element of $\mathbb{N}$, and $d, p, q, r$ be real numbers. Suppose $1 \leq i \leq m$ and $|y-x|<d$ and $|z-x|<d$ and $p=(\operatorname{proj}(i, m))(y)$ and $z=(\operatorname{reproj}(i, y))(q)$ and $r \in[p, q]$ and $w=(\operatorname{reproj}(i, y))(r)$. Then $|w-x|<d$.

(45) Let $m$ be a non empty element of $\mathbb{N}, f$ be a partial function from $\mathcal{R}^{m}$ to $\mathbb{R}, X$ be a subset of $\mathcal{R}^{m}, x, y, z$ be elements of $\mathcal{R}^{m}, i$ be an element of $\mathbb{N}$, and $d, p, q$ be real numbers. Suppose that $1 \leq i \leq m$ and $X$ is open and $x \in X$ and $|y-x|<d$ and $|z-x|<d$ and $X \subseteq \operatorname{dom} f$ and for every element $x$ of $\mathcal{R}^{m}$ such that $x \in X$ holds $f$ is partially differentiable in $x$ w.r.t. $i$ and $0<d$ and for every element $z$ of $\mathcal{R}^{m}$ such that $|z-x|<d$ holds $z \in X$ and $z=(\operatorname{reproj}(i, y))(p)$ and $q=(\operatorname{proj}(i, m))(y)$. Then there exists an element $w$ of $\mathcal{R}^{m}$ such that $|w-x|<d$ and $f$ is partially differentiable in $w$ w.r.t. $i$ and $f_{z}-f_{y}=(p-q) \cdot \operatorname{partdiff}(f, w, i)$.

(46) Let $m$ be a non empty element of $\mathbb{N}, h$ be a finite sequence of elements of $\mathcal{R}^{m}, y, x$ be elements of $\mathcal{R}^{m}$, and $j$ be an element of $\mathbb{N}$. Suppose len $h=$ $m+1$ and $1 \leq j \leq m$ and for every natural number $i$ such that $i \in \operatorname{dom} h$ holds $h_{i}=\left(y\left\lceil\left((m+1)-^{\prime} i\right)\right) \frown\langle\underbrace{0, \ldots, 0}_{i-^{\prime} 1}\rangle\right.$. Then $x+h_{j}=\left(\operatorname{reproj}\left((m+1)-^{\prime}\right.\right.$ $\left.\left.j, x+h_{j+1}\right)\right)\left(\left(\operatorname{proj}\left((m+1)-^{\prime} j, m\right)\right)(x+y)\right)$.

(47) Let $m$ be a non empty element of $\mathbb{N}, f$ be a partial function from $\mathcal{R}^{m}$ to $\mathcal{R}^{1}, X$ be a subset of $\mathcal{R}^{m}$, and $x$ be an element of $\mathcal{R}^{m}$. Suppose that

(i) $X$ is open,

(ii) $\quad x \in X$, and 
(iii) for every element $i$ of $\mathbb{N}$ such that $1 \leq i \leq m$ holds $f$ is partially differentiable on $X$ w.r.t. $i$ and $f \uparrow^{i} X$ is continuous on $X$.

Then

(iv) $\quad f$ is differentiable in $x$, and

(v) for every element $h$ of $\mathcal{R}^{m}$ there exists a finite sequence $w$ of elements of $\mathcal{R}^{1}$ such that $\operatorname{dom} w=\operatorname{Seg} m$ and for every element $i$ of $\mathbb{N}$ such that $i \in$ Seg $m$ holds $w(i)=(\operatorname{proj}(i, m))(h) \cdot \operatorname{partdiff}(f, x, i)$ and $f^{\prime}(x)(h)=\sum w$.

(48) Let $m$ be a non empty element of $\mathbb{N}, f$ be a partial function from $\left\langle\mathcal{E}^{m},\|\cdot\|\right\rangle$ to $\left\langle\mathcal{E}^{1},\|\cdot\|\right\rangle, X$ be a subset of $\left\langle\mathcal{E}^{m},\|\cdot\|\right\rangle$, and $x$ be a point of $\left\langle\mathcal{E}^{m},\|\cdot\|\right\rangle$. Suppose that

(i) $X$ is open,

(ii) $\quad x \in X$, and

(iii) for every element $i$ of $\mathbb{N}$ such that $1 \leq i \leq m$ holds $f$ is partially differentiable on $X$ w.r.t. $i$ and $f \uparrow^{i} X$ is continuous on $X$.

Then

(iv) $\quad f$ is differentiable in $x$, and

(v) for every point $h$ of $\left\langle\mathcal{E}^{m},\|\cdot\|\right\rangle$ there exists a finite sequence $w$ of elements of $\mathcal{R}^{1}$ such that $\operatorname{dom} w=\operatorname{Seg} m$ and for every element $i$ of $\mathbb{N}$ such that $i \in \operatorname{Seg} m$ holds $w(i)=(\operatorname{partdiff}(f, x, i))(\langle(\operatorname{proj}(i, m))(h)\rangle)$ and $f^{\prime}(x)(h)=$ $\sum w$.

(49) Let $m$ be a non empty element of $\mathbb{N}, f$ be a partial function from $\left\langle\mathcal{E}^{m},\|\cdot\|\right\rangle$ to $\left\langle\mathcal{E}^{1},\|\cdot\|\right\rangle$, and $X$ be a subset of $\left\langle\mathcal{E}^{m},\|\cdot\|\right\rangle$. Suppose $X$ is open. Then for every element $i$ of $\mathbb{N}$ such that $1 \leq i \leq m$ holds $f$ is partially differentiable on $X$ w.r.t. $i$ and $f \uparrow^{i} X$ is continuous on $X$ if and only if $f$ is differentiable on $X$ and $f_{\uparrow X}^{\prime}$ is continuous on $X$.

\section{REFERENCES}

[1] Grzegorz Bancerek. The fundamental properties of natural numbers. Formalized Mathematics, 1(1):41-46, 1990.

[2] Grzegorz Bancerek and Krzysztof Hryniewiecki. Segments of natural numbers and finite sequences. Formalized Mathematics, 1(1):107-114, 1990.

[3] Czesław Byliński. Finite sequences and tuples of elements of a non-empty sets. Formalized Mathematics, 1(3):529-536, 1990.

[4] Czesław Byliński. Functions and their basic properties. Formalized Mathematics, 1(1):5565, 1990.

[5] Czesław Byliński. Functions from a set to a set. Formalized Mathematics, 1(1):153-164, 1990.

[6] Czesław Byliński. Partial functions. Formalized Mathematics, 1(2):357-367, 1990.

[7] Agata Darmochwał. The Euclidean space. Formalized Mathematics, 2(4):599-603, 1991.

[8] Noboru Endou and Yasunari Shidama. Completeness of the real Euclidean space. Formalized Mathematics, 13(4):577-580, 2005.

[9] Noboru Endou, Yasunari Shidama, and Keiichi Miyajima. Partial differentiation on normed linear spaces $\mathcal{R}^{n}$. Formalized Mathematics, 15(2):65-72, 2007, doi:10.2478/v10037007-0008-5.

[10] Krzysztof Hryniewiecki. Basic properties of real numbers. Formalized Mathematics, 1(1):35-40, 1990. 
[11] Hiroshi Imura, Morishige Kimura, and Yasunari Shidama. The differentiable functions on normed linear spaces. Formalized Mathematics, 12(3):321-327, 2004.

[12] Takao Inoué, Noboru Endou, and Yasunari Shidama. Differentiation of vector-valued functions on $n$-dimensional real normed linear spaces. Formalized Mathematics, 18(4):207-212, 2010, doi: 10.2478/v10037-010-0025-7.

[13] Jarosław Kotowicz. Real sequences and basic operations on them. Formalized Mathematics, 1(2):269-272, 1990.

[14] Jarosław Kotowicz. Functions and finite sequences of real numbers. Formalized Mathematics, 3(2):275-278, 1992.

[15] Yatsuka Nakamura, Artur Korniłowicz, Nagato Oya, and Yasunari Shidama. The real vector spaces of finite sequences are finite dimensional. Formalized Mathematics, 17(1):19, 2009, doi:10.2478/v10037-009-0001-2.

[16] Takaya Nishiyama, Keiji Ohkubo, and Yasunari Shidama. The continuous functions on normed linear spaces. Formalized Mathematics, 12(3):269-275, 2004.

[17] Beata Padlewska and Agata Darmochwał. Topological spaces and continuous functions. Formalized Mathematics, 1(1):223-230, 1990.

[18] Konrad Raczkowski and Paweł Sadowski. Topological properties of subsets in real numbers. Formalized Mathematics, 1(4):777-780, 1990.

[19] Walter Rudin. Principles of Mathematical Analysis. MacGraw-Hill, 1976.

[20] Laurent Schwartz. Cours d'analyse. Hermann, 1981.

[21] Wojciech A. Trybulec. Vectors in real linear space. Formalized Mathematics, 1(2):291-296, 1990.

[22] Zinaida Trybulec. Properties of subsets. Formalized Mathematics, 1(1):67-71, 1990.

[23] Edmund Woronowicz. Relations defined on sets. Formalized Mathematics, 1(1):181-186, 1990.

[24] Hiroshi Yamazaki, Yoshinori Fujisawa, and Yatsuka Nakamura. On replace function and swap function for finite sequences. Formalized Mathematics, 9(3):471-474, 2001.

Received April 22, 2010 